\title{
Weitere Beiträge zur Lehre von der Wendung auf einen Fuss.
}

\author{
Von \\ Dr. med. W. Nagel, \\ Privatdocent für Geburtshülfe und Gynäkologie an der Königl. Friedrich-Wilhelm-Universität \\ zu Berlin. \\ Vorgetragen in der geburtshülflichen Gesellschaft zu Edinburgh \\ am 9. November 1892.
}

Es ist allgemein bekannt - und wird besonders hier in Edinburgh noch in lebhafter Erinnerung sein, - welchen wichtigen Antheil an der Einführung der Wendung auf den oberen Fuss Sir James Simpson gehabt hat und seine Auseinandersetzung für die Vorzüge des oberen Fusses in schwierigen Wendungsfällen war so treffend, dass viele angesehene Geburtshêlfer noch heute rathen, ausschliesslich auf den oberen Fuss zu wenden. An Widersprüchen hiergegen hat es indessen nicht gefehlt, so hat unter späteren Autoren besonders Galabin ${ }^{1}$ ) in London in scharfsinniger Weise die Vorzüge dargelegt, welche das Ergreifen des unteren Fusses bieten und es muss wohl heut zu Tage als eine feststehende Thatsache angesehen werden, dass es für das Gelingen der Umdrehung des Kindes in utero ganz gleichgiltig ist, welches Bein man ergreift. Die theoretischen Erwägungen, die Versuche am Phantom und die klinischen Erfahrüngen dor letzten Jahre sind zu dem übereinstimmenden Ergebnisse gekommen, welches ich auch

1) A. L. Galabin. On the choice of the leg which should be seized in version for presentation of the upper extremity. Transactions of the obstetrical Society of London. Vol. XIX. 1877. p. 239. 
aus eigener an 94 Querlagen (bei 19 stand die Blase z. Zeit der Wendung, 9 waren in Verbindung mit Placenta praevia) gewonnener klinischer Erfahrung bestätigen kann, dass die Umwandlung der Querlage in eine Geradlage sich gleich gut auf das obere wie auf das untere Bein bewerkstelligen lässt.

Jeder erfahrene Geburtshelfer wird wohl indessen zuweilen einen Fall erlebt haben, wo kräftige Wehen die vorliegende Schulter tief in das Becken hineingepresst haben und wo die Umdrehung des Kindes, gleichgültig welches Bein man zuerst genommen hat, erst dann gelingt, wenn man auch das zweite Bein herunterholt. In den letzten Jahren hat G. E. Herman') 3 Fälle von starker Zusammenziehung des unteren Uterinsegments um die vorliegende Schulter als Hinderniss für die Umđrehung des Kindes selbst nach Herunterholen beider Füsse beschrieben. Ich selbst habe vor einigen Monaten in Berlin einen ähnlichen ausgesprochenen Fall erlebt, der eine weitere Stufe des von Herman beschriebenen Zustandes darstellt, weshalb ich denselben hier mittheilen möchte, zumal, wie Galabin und Champneys ${ }^{2}$ ) ebenfalls betonen, eine Ausziehung des unteren Uterinsegments, als Hinderniss für die Wendung bei Querlagen, heut za Tage im Ganzen ein seltenes Ereigniss ist. Es handelte sich in meinem Falle um eine 41 jährige Mehrgebärende am normalen Ende der Schwangerschaft, wo der rechte Arm gleichzeitig mit dem Blasensprunge vorfiel; es stellten sich sofort sehr heftige Wehen ein, die Schlag auf Schlag folgten und die alsbald in starke Presswehen übergingen. Da es Nacht und die Entfernung eine grosse war, so erreichte die Meldung der Hebamme mich erst 4 Stunden nach dem Blasensprunge. Ich eilte sofort hin und fand die Frau in sehr heftigen Wehen mit normaler Temperatur und einem Puls von 76-80; der Contractionsring war sehr stark ausgeprägt, in Nabelhöhe zu sehen und zu fühlen, das Kind lag, wie deullich (nach Einleitung der Chloroformnarcose) durch die dünnen Bauchdecken zu sehen und durch die äussere Untersuchung allein festzustellen, in dem stark ausgedehnten unteren Uterinsegment, das eigentliche Corpus uteri sass wie eine Halbkugel rechts oben dem verdünnten Uterinsegmente auf; der

1) G. E. Herman (London). Note on one of the causes of difficulty in turning, with remarks on the practice of amputating the procident arm. Transactions of the obstetrical Society of London. Vol. XXVIII. 1886. p. 150 .

2) Transact. of the obst. Soc. of London. Vol, XXVIII. 1886, p. 153. 
vorgefallene Arm war bis zur Schulter geboren, hinter dem Introitus vaginae kam man sofort auf den Thorax, der Kopf lag auf der rechten Darmbeinschaufel, der Rücken war nach hinten gekehrt. Dã ich mich durch Eindrücken des Epigastriums am Kinde ron dem völligen Stillstande des Herzens äberzeugen konnte, so nahm ich, der bedrohlichen Erscheinungen yon Seiten des Uterus wegen, von der Wendung Abstand und decapitirte das Kind, wonach die Entbindung leicht erfolgte. Das Wochenbett war ungestört.

Eine Reihe hervorragender Geburtshelfer früherer und späterer Jahre (Baudelocque, Lachapelle, Hohl, Gusserow u. A.) gingen in der Wahl des Fusses nicht von dem Gesichtspunkte aus, die günstigste Handhabe für die Umdrehung des Kindes zu gewinnen, sondern ergriffen dasjenige Bein, welches unmittelbar nach vollzogener Wendung vorn hinter die Symphyse kömmt, indem sie der bekannten Thatsache Rechnung trugen, dass die Geburt eines ausgetragenen Kindes in unvollkommener Fusslage nur dann vor sich gehen kann, wenn das ausgestreckte Bein vorn hinter der Symphyse liegt. In Folge dieser Ueberlegung ziehen die genannten Autoren bei dorso-posterioren Lagen den oberen Fuss vor, während sie bei dorso-anterioren Lagen den unteren Fuss wählen.

Galabin ${ }^{1}$ ) and Fritsch ${ }^{2}$ ) meinen indessen, dass bei dorsoposterioren Lagen, wenn der obere Fuss genommen worden ist, der Rücken des Kindes in vielen Fällen hinten bleibt, dass mit anderen Worten das heruntergeholte Bein unmittelbar nach vollzogener Wendung nicht vorn, hinter der Symphyse liegt, sondern sich dorthin erst drehen muss, so dass also der beabsichtigte Vortheil völlig verloren geht. Fritsch spricht allerdings nur vom Rücken, nicht von den Füssen; er giebt nicht an, wo in seinen 14 Fällen das ergriffene Bein nach vollzogener Wendung stand, ob vorn hinter der Symphyse oder in der Kreuzbeinhöhlung. Ga labin spricht ebenfalls nür vom Rücken und thut der Lage des ergriffenen Beines nach der Wendung keine Erwähnung. Ich nehme indessen an, dass die beiden Verfasser mit der Stellung des Rückens die Lage des ausgestreckten Beines anzugeben beabsichtigen; denn es ist ganz gleichgiltig, ob der Rücken nach hinten

1) Galabin a. a. 0. (1. c.).

2) Fritsoh. Zur Lehre rom Ergreifen der Füsse bei der Wendung. Dieses Archiv Bd. IV. 
gerichtet ist, wenn nicht gleichzeitig das ausgestreckte Bein hinten in der Kreuzbeinhöhlung liegt. Fritsch und Galabin stützen sich auf klinische Erfahrungen und ein Zweifel an die Richtigkeit der Beobachtungen dieser beiden verdienten Forscher ist nicht zulässig. Ich darf aber in Bezug auf die Genauigkeit meiner Beobachtungen dasselbe Recht beanspruchen und diese stehen mit den Angaben der beiden Forscher in Widerspruch. In 19 Fällen von dorso-posterioren Querlagen, wo ich in Uebereinstimmung mit der Lehre Gusserow's das obere Bein ergriffen habe, ist dieses ohne Ausnahme unmittelbar nach der Wendung vorn hinter die Symphyse gekommen und auch dort geblieben. Der Widerspruch zwischen den Galabin-Fritsch'schen und den meinigen Beobachtungen ist nicht leicht zu erklären, doch scheint mir, dass die von Galabin gegebene Auseinandersetzung hierfür sehr wohl zu verwenden ist. Galabin (a. a. O. S. 246) sagt nämlich: „in abdomino-anterior positions, however, the pubes allows but scanty space for the hand to be bent backwards round the sharp angle at the concave side of the pelvic curve. Hence the hand naturally deviates more or less to one side, and traction is not directed sufficiently forward with regard to the pelvis and the foetus. At the same time, by its lateral deviation, it tends to rotate the foetus about its antero-posterior axis". $\mathrm{Zu}$ dieser treffenden Erklärung möchte ich als Ergänzung zufügen, dass sie besonders für die Fälle passt, wo bei den gedachten Lagen der Steiss tief steht; in derartigen Fällen wird man, obwohl ich es selber bisher nicht erlebt habe, sicherlich am häufigsten beobachten können, dass das ergriffene Bein, selbst wenn es das obere ist, beim Anziehen einfach seitlich herunter gleitet, ohne dass die beabsichtigte Drehung des Kindes um seine Längsachse stattfindet. Steht dagegen der Steiss hoch (im Fundus uteri) so wird der Mechanismus der Umdrehung demjenigen bei Schädellage ähneln, nämlich eine Rotirung über die Bauchfäche des Kindes, um den queren bilateralen Durchmesser des kindlichen Körpers darstellen und das obere (der vorliegenden Schulter entgegengesetzte) Bein wird unmittelbar nach vollzogener Wendung vorn hinter die Symphyse kommen. Eine weitere Ursache für das Ergebniss in meinen Fällen mag darin liegen, dass ich, ebenfalls entsprechend den Lehren Gusserow's, die Frau auf diejenige Seite lagere, in welcher die Füsse liegen und wenn die Füsse rechts liegen die linke Hand (und umgekehrt) in den Uterus einführe. Es mag bei dieser 
Lagerung leichter sein, den Fuss während des Herunterziehens nach vorne zu führen und dadurch das Kind um seine Längsachse zu rotiren.

In der Mehrzahl der Fälle von dorso-anterioren Lagen wird das untere Bein unmittelbar nach vollzogener Wendung vorn hinter die Symphyse kommen. Eine Ausnahme hiervon bilden jedoch diejenigen (dorso-anterioren) Lagen, wo der Steiss im Fundus steht und der Mechanismus in der Umdrehung des. Kindes demjenigen bei Schädellage ähnelt; in solchen Fällen wird man; wie ich an der Lebenden beobachtet habe und wie Jeder sich ausserdem am Phantom überzeugen kann, beim Ergreifen des der vorliegenden Schulter entsprechenden Beines manchmal erleben, dass dasselbe nach vollzogener Wendung hinten in der Kreuzbeinhöhlung liegt und sich also nun nach vorn drehen muss. Nehmen wir zum Beispiel eine I. dorso-anteriore Querlage; liegt in solchem Falle der Steiss tief, so wird das ergriffene untere Bein sofort hinter die Symphyse kommen. Steht aber, wie es in praxi nicht selten vorkommt, in der gedachten Lage der Steiss hoch im Fundus, so ist die Lage, mit Rücksicht auf die Entstehung derselben, als eine II. Schädellage aufzufassen, bei welcher der Kopf so stark nach links abgewichen ist, dass nicht dieser, sondern die Schulter den vorliegenden Theil bildet; wie bei II. Schädellage, so steht in der gedachten Lage der Steiss rechts oben vorne, die Füsse links oben hinten. Will man nun aus II. Schädellage in der Weise wenden, dass das ergriffene Bein nach der Wendung vorn, hinter die Symphyse kommt, so muss man das linke Bein herunterholen; in dem gedachten Falle, wenn wir denselben als Querlage betrachten (und das müssen wir von einem practischen Standpunkte aus), ist aber das linke Bein das obere. Zieht der Operateur, wie ich es in praxi gethan habe, dieses obere Bein herunter, so wird es nach vollzogener Wendung, analog dem Mechanismus bei II. Schädellage, vorn hinter die Symphyse kommen. Nimmt der Operateur dagegen, in Uebereinstimmung mit der Lehre, dass man bei dorso - anterioren Querlagen den unteren Fuss (das heisst den der vorliegenden Schulter entsprechenden) wählen soll, in dem "gedachten Falle diesen, also den rechten, so wird er, wie ich in praxi gesehen habe (siehe den Fall No. 23, vergl. No. 29) und wie sicherlich auch Andere gesehen, haben werden, erleben, dass das ergriffene (hier also das rechte) Bein unmittelbar nach der Wendung hinten in der Kreazbeinhöhlung steht, indem das 
Kind, auf Anziehen des Fusses, sich analog dem Mechanismus bei II. Schädellage gedreht hat.

$\mathrm{Ob}$ in der gedachten Lage die Umdrehung des Kindes in der eben beschriebenen Weise geschehen wird, oder so, dass das ergriffene (untere) Bein unmittelbar nach der Wendung hinter die Symphyse kommt, wird meiner Ansicht nach theils von der Biegsamkeit des Kindes, theils' von dem Grade der Zusammenziehung des Uterus um den kindlichen Körper abhängen. Die Bedeutung des erstgenannten Factors für die Umdrehung des Kindes hat Galabin in seiner angeführten Arbeit eingehend beleuchtet und es ist klar, dass in dorso-anterioren Lagen mit im Fundus stehenden Steiss das untere (der vorliegenden Schulter entsprechende) Bein nur dann der seitlichen Uteruswand entlang nach vorn hinter die Symphyse gezogen werden kann, wenn die Biegsamkeit des kindlichen Körpers eine so grosse ist, dass er über seine rechte oder linke Seite gewissermaassen zusammengeklappt werden kann. Dieser Grad ron Biegsamkeit wird besonders dann eintreten, wenn der Tonus des Körpers aufgehoben ist, wenn also das Kind todt oder in Lebensgefahr sich befindet. Es ist ferner klar, dass diese letztgenannte Art der Umdrehung mehr Platz fordert, als die weiter oben beschriebene. Auf Grund dieser Auseinandersetzung, welche sich mit meinen Beobachtungen an der Lebenden deckt, meine ich, dass es bei dorso-anterioren Lagen mit hochstehendem Steiss keineswegs als feststehende Regel gilt, dass das untere (der vorliegenden Schulter entsprechende) Bein unmittelbar nach der Wendung vorn hinter die Symphyse kommt.

In allen Fällen von Wendung auf das obere Bein bei dorsoanterioren Lagen, mit Ausnahmeder soeben besprochenen, wird der Rücken unmittelbar nach vollzogener Wendung zunächst hinten stehen und hier decken sich meine klinischen Beobachtungen mit denen von Fritsch und Galabin, indem in den Fällen, wo ich bei dorso-anterioren Lagen nach den Vorschriften von Barnes auf den oberen Fuss wendete, das heruntergeholte Bein, und somit auch der Rücken, nach vollzogener Wendung hinten in der Kreuzbeinhöhlung lag. Wendet man bei dorso-posterioren Lagen auf das untere (der vorliegenden Schulter entsprechende) Bein, so wird dieses ebenfalls als Regel nach vollzogener Wendung hinten in der Kreuzbeinhölung stehen. In meinen Wendungsfällen der letztgenannten Art habe ich keine Ausnahme von dieser Regel gesehen und eine Ausnahme ist wohl nur dann möglich, 
wenn schon innerhalb des Uterus während der Umdrehung diejenige Rotation um die Längsachse des Körpers geschieht, welche sonst immer auf einem späteren Stadium der Geburt, nämlich nach Herstellung der Geradlage, stattfindet. Ich bedaure, dass Galabin in seiner ausgezeichneten Arbeit diese Thatsache nicht erwähnt und die jst doch eine der hauptsächlichsten Einwendungen vieler Geburtshelfer gegen das Ergreifen des unteren Fusses bei dorso-posterioren Lagen. Es ist ja ganz richtig, dass das heruntergeholte Bein auch in diesen Fällen schliesslich das vordere wird, worauf Galabin mit Recht seinen (auch von Gusserow u. A. gegebenen) Rath, den vorgefallenen Arm anzuschlingen, damit man bei der Ausziehung des Kindes keine Schwierigkeit mit der Lösung des vorderen Armes haben soll, gründet; zuvor muss aber der kindliche Rumpf die unten zu besprechende Drehung um seine Längsachse machen.

Es ist jetzt eine allgemein anerkannte, von Wiegand und Braxton Hicks') seiner Zeit besonders hervorgehobene Thatsache, dass das Kind in den meisten Fällen pon sogenannter Querlage schräg liegt und nicht quer, dass entweder der Kopf oder der Steiss dem Os näher ist. Welcher Theil der tiefstehende ist und welcher Mechanismus bei der Umdrehung in dem gegebenen Falle obwalten wird, das ist, wie ich betonen möchte, davon abhängig, aus welcher ursprünglichen Lage (Schädel- oder Steisslage) die Schräglage entstanden ist und es steht fest, dass man manchmal nach Wendungen unter den oben besprochenen Verhältnissen, besonders wenn man bei dorso-anteriorer Lage den oberen und bei dorso-posterioren Lagen den unteren Fuss ergreift, zunächst eine unvollkommene Fusslage mit ausgestrecktem hinteren Bein vor sich hat. Da das Kind in dieser Lage nicht geboren werden kann - ich sehe von unreifen und macerirten Kindern ab -, so frägt sich, was nun weiter geschehen soll.

Es ist ja, wie gesagt, eine Thatsache, dass das heruntergeschlagene Bein, selbst wenn es auch Anfangs hinten in der Kreuzbeinhöhlung liegt, im Verlaufe der Geburt nach kürzerem oder längerem Zuwarten sich schliesslich nach vorn dreht. In welcher Weise diese Drehung stattfindet und wie man dieselbe erentuell za begünstigen rermag, darüber gehen die An-

1) J. Braxton Hicks. On combined external and internal Version. London 1864. 
sichten der Geburtshelfer aller Zeiten sehr auseinander, so dass ich eine einheitliche und brauchbare Anweisung weder in den Lehrbüchern noch in den Originalarbeiten über dieses Capitel gefunden habe. Einen Auszug der Literatur habe ich trüher gegeben und verweise darauf. Ausser den dort wieder gegebenen Ansichten von Lachapelle, Credé, Fritsch, Küstner, die sich am meisten mit dieser Frage beschäftigt haben, ist mir nachträglich noch folgende Aeusserung Credé's zu Gesicht gekommen; Seite 713 des früher schon angeführten Werkes (Klinische Vorträge über Geburtshülfe, Berlin 1854) sagt er bei Besprechung der Drehungen des Kindes: „Dabei (bei allen unvollkommenen Fuss- und Knielagen, wo das herabgeschlagene Bein das falsche hintere ist) stellt sich heraus, dass nicht die gewöhnlichen Uebergänge aus der vierten in die erste Stellung, aus der dritten in die zweite von Statten gehen, sondern dass die erste Stellung sich in die zweite, die zweite in die erste, ferner die dritte in die vierte, die vierte in die dritte umdrehen, letztere vierte und dritte Stellungen können dann schliesslich noch in die erste und zweite übergehen. Dieselben Drehungen haben wir in den hierher gehörigen Fällen künstlich zu machen u. s. w." Credé nimmt also ebenfalls an, dass in gewissen Fällen der Rücken sich auf dem kürzeren Wege drehe und ân der Symphyse vosbeigleite. Ich gebe zu, dass diese Frage in praxi zuweilen von untergeordneter Bedeutung sein mag. Es muss aber die Aufgabe einer rationellen Geburtshülfe sein, möglichst genaue Kenntnisse über die einzelnen Vorgänge, welche sich bei der natürlichen Geburt des Kindes abspielen, zu erwerben, damit man, wenn Hülfe nöthig, dem von der Natur eingeschlagenen Weg folgen kann. Denn selbst wenn man, wie ich es ebenfalls thue, die Regel festhält, nach der Wendung die weitere Geburt zunächst den Wehen zu überlassen, so werden doch jedem Geburtshelfer Fälle vorkommen, wo man die Entbindung möglichst beschleunigen muss.

Meine Beobachtungen verfolgten nun den Zweck, bei unvollkommener Fusslage mit ausgestrecktem hinteren Bein die Art und Weise festzustellen, in welcher der kindliche Rumpf um seine Längsachse sich dreht, damit das ausgestreckte Bein vorn hinter die Symphyse komme und ich glaube aus den eben erwähnten Gründen, dass sie ein gewisses Interesse von Seiten der Fachgenossen beanspruchen dürfen. Die Fälle stammen alle aus der geburtshülflichen Universitäts - Poliklinik der Königl. Charité in Berlin, die ich damals als Assistenzarzt leitete, und ich bin Herrn 
Professor Dr. Gusserow für die Ueberlassung des Materials zu Dank verpflichtet.

Ich verfüge über 30 derartige Fälle, wo ich entweder den hinteren Fuss vorgefallen fand oder wo ich aus verschiedenen Gründen den hinteren Fuss herunterholte und von welchen ich mir während und nach der Geburt genaue Aufzeichnungen gemacht habe. 24 mal bin ich bei der Vollendung der Geburt zugegen gewesen und habe selber die Drehung gesehen, 6 mal war ich daran verhindert und die Drehung ist in diesen 6 Fällen von dem Practikanten beobachtet worden. Ich betone, dass in sämmtlichen Fällen die Geburt nach der Wendung zunächst der Natur überlassen worden ist, so dass die beobachtete Drehung also einen natürlichen Vorgang darstellt. Wenn im späteren Verlaufe der Geburt ein Zug an dem Bein ausgeübt worden ist, so ist dieses jedesmal in der Geburtsgeschichte (siehe unten) angefühırt worden. 10 Fälle betrafen Placenta praevia, welche nach Braxton Hicks behandelt worden sind, indem ich möglichst früh die combinirte Wendung ausführte und dann die Ausstossung des Kindes den Wehen überliess; in diesen Fällen ist, der Blutstillung halber mittels einer Schlinge ein so starker Zug an dem ausgestreckten Bein ausgeübt worden, wie das Gewicht des Armes allein zu erzeugen vermag und ich glaube nicht, dass ein solcher mässiger Zug den natürlichen Drehungsvorgang zu beeinflussen im Stande ist.

Die ersten 18 Beobachtungen finden sich in diesem Archiv, Bd. 39, 1891; die übrigen lasse ich hier folgen.

Fall 19. 21. März 1891. K., 35jähr. VIII para. Blase vor 12 Stunden gesprungen bei mangelhaft erweitertem Muttermunde; I, unvollkommene Fusslage, der rechte hintere Fuss ragte zur Vulva heraus, Muttermund fast verstrichen, in demselben lag der andere Fuss und eine Nabelschnurschlinge, welche während der Wehen pulslos wurde; aus diesem Grunde wurde die Geburt beschleunigt, indem ich unter gleichzeitigem Drucke auf den Fundus, während der Wehen mässig an dem vorgefallenen Fuss zog; dabei drehte sich der ursprünglich nach links gekehrte Rücken dem Promontorium vorbei in die rechte Beckenhälfte, so dass eine II. unyollkommene Fusslage entstand und das ausgestreckte Bein lag nun vorn hinter der Symphyse. Nachdem der Steiss durchgeschnitten, wurde das ausgetragene, gut entwickelte Kind ạusgezogen und vollkommen wiederbelebt. Beide Arme waren hochgeschlagen und mussten gelöst werden.

Fall 20. 28. October 1891. W., 33 jähr. IV para. Erster Zwilling natürlich in Schädellage geboren, zweiter Zwilling lag in II. Fusslage mit Vorliegen des linken hinteren Fusses. Blase stand: ich sprengte dieselbe, der vorliegende Fuss trat sofort tiefer und unter einigen kräftigen Wehen drehte sich das Kind mit dem Rücken dem Promontorium 
vorbei in die linke Beckenhälfte, so dass das ausgestreckte (linke) Bein vorn hinter die Symphyse kam; das lebende, ausgetragene Kind (Länge 47 , Kopfumfang $34 \mathrm{~cm}$ ) wurde in I. unvollkommener Fusslage leicht und schnell geboren.

Fall 21. 3. December 1891. St., 31jähr. IIIpara. Blase vor 36 Stunder bei engem Muttermunde gesprungen, gleichzeitig ging Meconium ab. I. Steisslage, Herztöne 120-140, schwach. Da der Sttiss bei meiner Ankunft trotz der langen Daner der Geburt nur mit einem kleinen Segment durch den Beckeneingang getreten war, so holte ich, bei handtellergrossem Muttermunde, das im Kniegelenke ausgestreckte rechte Bein herunter; dasselbe lag alsdann hinten in der Kreuzbeinhöhlung. Der Rücken, welcher ursprünglich links seitlich stand, drehte sich unter kräftigen Wehen dem Promontorium vorbei in die rechte Beckenhälfte, so dass das ausgestreckte Bein vorn hinter die Symphyse kam. Die weitere Geburt vollzog sich in II. unvollkommener Fusslage; nachdem das Kind bis zum Nabel geboren worden war, zog ich es, während gleichzeitig ein sehr kräftiger Druck von aussen auf den Kopf ausgeübt wurde, aus, was des engen Beckens und des sehr grossen Kindes wegen schwierig war. Das Kind (Länge 55, Kopfumfang $38 \mathrm{~cm}$ ) war todt.

Fall 22. 12. December 1891. S., ca. 35jähr. VIIpara. II. Steisslage; Placenta praevia centralis. Muttermund dreimarkstückgross. Grosse Blutung. Darchbohrung der Placenta mit zwei Fingern in der Nähe der Nabelschnurinsertion, ich holte das linke Bein herunter und legte es in eine Schlinge; dasselbe lag alsdann hinten in der Kreuzbeinhöhlung. Unter guten Wehen drehte sich das ausgetragene Kind mit dem Rücken dem Promontorium vorbei in die linke Beckenhälfte, so dass das ausgestreckte Bein vorn hinter die Symphyse kam, und wurde 6 Stunden später in I. unvollkommener Fusslage leicht aber todt geboren.

Fall 23. 20. December 1892. G., 24jähr. IIIpara. I. Querlage (aus II. Schädellage entstanden, Steiss im Fundus, Füsse links oben hinten, Rücken vorn) mit Vorfall des rechten Armes, welcher angeschlungen wurde. Ich holte das der vorliegenden Schulter entsprechende Bein (das rechte) herunter; dasselbe lag nach vollzogener Wendung hinten in der Kreuzbeinhöhlung, der Rücken stand links hinten. Bei guten Wehen drehte das Kind sich alsbald, unter gleichzeitigem leichten Anziehen des Fusses meinerseits, mit dem Rücken dem Promontorium vorbei in die rechte Beckenhälfte, so dass das ausgestreckte Bein vorn hinter die Symphyse kam. Unter kräftigen Wehen erfolgte die weitere Geburt leicht in II. unvollkommener Fusslage. Das ausgetragene Kind (Länge 48 , Kopfumfang $33^{1} / 2 \mathrm{~cm}$ ) lebte.

Fall 24. 5. Januar 1892. G., 27 jähr. IV para. Hängebauch. Zwillinge. Blase vor $2 \mathrm{St}$. gesprungen. Muttermund über fünfmarkstückgross. II. Steisslage. Da der Steiss noch beweglich über dem Beckeneingange stand, so holte ich das linke Bein herunter und streckte es aus; dasselbe lag alsdann hinten in der Kreuzbeinhöhlung; der Rücken, welcher zunächst rechts hinten stand, drehte sich alsbald mit dem Rücken dem Promontorium vorbei in die linke Beckenhälfte, so dass das ausgestreckte Bein vorn hinter die Symphyse kam. Die weitere Geburt des ausgetragenen kräftigen lebenden Kindes erfolgte natürlich und leicht in I. unvollkommener Fusslage. (Zweites Zwillingskind natürlich in I. Schädellage geboren). 
Fall 25. 6. Januar 1892. S., 27jähr. IV para. Frühgeburt im IX. Monate, I. Querlage. Muttermund dreimarkstückgross, Placenta prae: via centralis. Grosse Blutung. Combinirte Wendung nach Braxton Hicks unter Durchbohrung der Placenta nahe am Rande mit zwei Fingern. Ich holte das linke Bein herunter, dasselbe lag nach vollzogener Wendung hinten in der Kreuzbeinhöhlung, der Rücken stand rechts seitlich. Unter kräftigen Wehen drehte sich das Kind mit dem Rücken dem Promontorium vorbei in die I. unvollkommene Fusslage und wurde in dieser Lage, mit dem ausgestreckten Bein vorn, 2 Stunden später natürlich aber todt geboren.

Fall 26. 16. Januar 1892. A., 34jähr. VIpara. Frühgeburt im IX. Monate. Starke Blutung seit 2 Stunden. I. Schädellage. Muttermund dreimarkstückgross, vorn rechts in demselben ist Placentargewebe zu fühlen. Combinirte Wendung nach Braxton Hicks unter Sprengung der Blase. Tch holte das zunächst erreichbare (linke) Bein herunter, dasselbe lag nach vollzogener Wendung hinten in der Kreuzbeinhöhlung, der Rücken stand rechts seitlich. Das Kind drehte sich alsbald während einer kräftigen Wehe in die linke Beckenhälfte, wobei der Rücken dem Promontorium vorbei glitt; das ausgestreckte Bein lag alsdann vorn hinter der Symphyse. 2 Stunden später wurde das Kind in I, unvollkommener Fusslage. leicht und lebend geboren.

Fall 27. 19. Januar 1892. M., 32jähr. Ipara. I. Steisslage. Beginn der Wehen vor 30 Stunden, Fruchtwasser vor 12 Stunden abgeflossen. Trotz kräftiger Wehen steht der Steiss noch im Beckeneingange, beweglich. Contractionsring 4 Querfinger oberhalb der Symphyse. Enges Becken (Spinae 24, Cristae 25, Conjugata externa $17 \frac{1}{2} \mathrm{~cm}$ ). Kindliche Herztöne 180. Ich holte das im Kniegelenke ausgestreckte rechte Bein herunter; dasselbe lag alsdann hinten in der Kreuzbeinhöhlang; der Rücken stand links rorn. Nach kurzem Zuwarten drehte sich der Rücken unter kräftigen Wehen nach hinten links, dann dem Promontorium vorbei in die rechte Beckenhälfte, so dass das ausgestreckte Bein vorn hinter die Symphyse kam. Da die kindlichen Herztöne auf 90 gesunken waren, so zog ich das Kind in der in erwähnter Weise entstandenen II. unvollkommenen Fusslage aus; die Lösung des hinteren Armes war besonders schwierig. Unter sehr kräftigem Druck von aussen wurde der Kopf durch den Beckeneingang gepresst und vollends mittels Mauriceau-Veit'schen Handgriffes entwickelt. Das ausgetragene Kind (Länge 49, Kopfumfang $34 \frac{1}{2} \mathrm{~cm}$ ) wurde vollkommen wiederbelebt, ein Dammriss II. Grades mit Seide genäht.

Fall 28. 4. Februar 1892. N., 32jähr. VIpara. Frühgeburt im Anfange des VIII. Monats. Blutet seit 3 Stunden erheblich. I. gemischte Steisslage; Muttermund fünfmarkstückgross, kein Placentargewebe zu fühlen. Ich sprenge die Blase und hole das rechte Bein herunter, welches alsdann hinten in der Kreuzbeinhöhlung lag. Während einer kräftigen Wehe und bei gleichzeitigem Druck auf den Fundus drehte sich der Rücken, welcher zunächst links hinten stand, dem Promontorium vorbei in die rechte Beckenhälfte und das ausgestreckte Bein lag alsdann vorn hinter der Symphyse. Leichte Ausziehung des todten Kindes in II. unvollkommener Fusslage. Die Placenta folgte unmittelbar, zu $1 / 3$ ihrer maternen Fläche mit festhaftenden Coagulis bedeckt, der Eihautriss befand sich dicht am Placentarrande.
Fall 29.
6. Februar 1892.
H., 32jähr. IVpara.
Hängebaxıch. 
Fruchtwasser vor 8 Stunden abgeflossen. Kindliche Herztöne 136, schwach. Muttermund vollkommen erweitert. Linke Schulter vorliegend. Rücken vorn, Kopf auf dem rechten Darmbeinschaufel, Steiss im Fundus links, Füsse rechts oben hinten (II. Querlage aus I. Schädellage entstanden). Ich holte das linke, der vorliegenden Schulter entsprechende Bein herunter, dasselbe lag nach vollzogener Wendung hinten in der Kreuzbeinhöhlung mit der grossen Zehe nach vorn, der Rücken stand rechts hinten. Alsbald drehte sich das Kind unter guten Wehen mit dem Rücken dem Promontorium vorbei in die linke Beckenhälfte, so dass das ausgestreckte (linke) Bein hinter die Symphyse $\mathrm{kam}$. Nach Durchschneiden des Steisses leichte Ausziebung in I. unvollkommener Fusslage. Der linke Arm war beim Herunterbolen des Beines vorgefallen uud vor Herstellung der Geradlage angeschlungen worden. Das kräftig entwickelte Kind (Länge $53^{1 / 2}$, Kopfumfang $36^{1 / 2} \mathrm{~cm}$ ) war asphyctisch, wurde aber vollkommen wiederbelebt.

Fall 30. 7. Februar 1892. F., 27jähr. III para. Frühgeburt im IX. Monate. Schwache Blutung seit 6 Tagen. Mattermund zweimarkstückgross, überall von Placentargewebe überdeckt. Combinirte Wendung nach Braxton Hicks unter Durchbohrung der Placenta am Rande derselben. Ich holte das linke Bein herunter, welches nach vollzogener Wendung hinten in der Kreuzbeinhöhlung lag, während der Rücken rechts stand. Das Kind drehte sich unter guten Wehen mit dem Rücken dem Promontorium vorbei in die linke Beckenhälfte und wurde 4 Stunden später in I. unvollkommener Fusslage, das ausgestreckte Bein vorn, natürlich und lebend geboren.

In sämmtlichen 30 Fällen fand die Drehung des kindlichen Rumpfes um seine Längsachse in der Weise statt, dass der Rücken dem Promontorium vorbeiglitt; das ursprünglich hinten in der Kreuzbeinhöhlung liegende Bein legte also, um nach vorn hinter der Symphyse zu kommen, stets den längeren Weg durch das Becken zurück, niemals den kürzeren Weg durch diejenige Beckenhälfte, in welcher der Rücken unmittelbar nach der Wendung stand. Bemerken will ich noch, dass alle die Kreissenden nach vollzogener Wendung in die Rückenlage gebracht wurden, und dass die Geburten in dieser Lage zu Ende gingen; ob die Seitenlage einen Einfluss auf die Drehung des Kindes auszuüben vermag, habe ich nicht versucht. Ich gehe weiter als Credé, Fritsoh, Küstner u. A., welche die Drehung mit dem Rücken dem Promontorium vorbei nur für gewisse Fälle zulassen und schliesse aus meinen Beobachtungen, dass die erwähnte Art der Drehung in Fällen von unvollkommener Fusslage mit ausgestrecktem hinteren Bein wahrscheinlich die einzige naturgemässe ist; überlässt man derartige Geburten der Natur, so werden die Wehen den kindlichen Rumpf stets unter der angeführten Drehung austreiben. 
Zu Gunsten meiner Annahme spricht ferner der Umstand, dass ich nur einmal eine Verdrehung des Kindes erlebt habe, indem der vordere Arm in diesem Falle (No. 8) in den Nacken geschlagen war; da es sich hierbei jedoch gleichzeitig um Vorfall einer schwach pulsirenden Nabelschnur handelte, welcher mich zwang, die Ausziehung trotz mangelhaft erweitertem Muttermunde zu beschleunigen, so muss es dahingestellt bleiben, ob letzteres nicht mit die Ursache für die regelwidrige Lage des Armes war.

In den letaten Jahren hat Berry Hart') die innere Drehung des Kindes etwas näher beleuchtet und sagt, dass derjenige Theil des foetalen Kopfes oder Körpers, welcher als vorangehender Theil die seitliche Hälfte des Sacralsegments berühre, nach vorne rotire. Er thut jedoch der unvollkommenen Fusslagen mit ausgestrecktem hinteren Bein keine Erwähnung; ich vermisse ferner in seinen Diagrammen über die Rotation den Weg durch die hintere BeckenhäIfte dem Promontorium vorbei. Das von Berry Hart aufgestellte Rotationsgesetz scheint mir auf die hier gedachten Lagen nicht zu passen.

Eine Erklärung für den etwas auffallenden Drehungsmechanismus würde die folgende sein, die aus den allgemein bekannten Geburtsfactoren hergeleitet ist: durch den nach hinten unten gerichteten Druck der Wehen bewegt sich der vorangehende Theil (das ausgestreckte Bein mit der entsprechenden Steisshälfte) dahin, wo das Becken am meisten Raum und folglich den geringsten Widerstand bietet, nämlich in die Kreuzbeinhöhlung; der Drehung des Rückens auf dem kürzeren Wege zur Symphyse, nämlich durch die entsprechende Beckenhälfte stellt der geringe Raum in der vorderen seitlichen Beckenhälfte für gewöhnliche Fälle (ich sehe von unreifen Früchten ab) ein unüberwindliches Hinderniss entgegen. Gleichzeitig mit der erwähnten Drehung tritt die vorangehende, hinten belegene, Steisshälfte tiefer; durch den Widerstand, welchen das hochgeschlagene Bein jetzt an der vorderen Beckenwand findet, wird es nun seinerseits in die Kreazbeinhöhlung hineingezwungen; wie allgemein bekannt, rotirt das ausgestreckte, hinten belegene Bein infolgedessen soweit nach vorne, bis der grösste Durchmesser des Steisses in dem geraden Duchmesser des Beckenausganges verläuft. Die einzige Ausnahme von dem hier

1) Berry Hart. On the nature and cause of the Movement of internal Rotation. Edinburgh Medical Journal 1886. 
beschriebenen Geburtsmechanismus habe ich in einem Falle von Placenta praevia im 7. Schwangerschaftsmonate erlebt, wo ich ebenfalls durch combinirte Wendung eine unvollkommene Fusslage mit ausgestrecktem hinteren Bein dargestellt hatte; um die Blutung zu stillen, musste etwas stärker am Beine des unreifen Kindes gezogen werden als gewöhnlich; die Drehung blieb indessen aus, indem das Kind nach genügender Erweiterung des Muttermundes in der genannten Stellung ausgezogen wurde. Bei Frühgeburten wird man wohl überhaupt öfters ein Ausbleiben der Drehung beobachten können, ohne dass dadurch die Gültigkeit des Seite 12 ausgesprochenen Satzes beeinträchtigt werden kann.

Das aus meinen Beobachtungen gewonnene Ergebniss möchte ich zum Schluss in folgenden Sätzen zusammenfassen:

Um die Umdrehung des Kindes in utero zu vollziehen, ist es ganz gleichgiltig, welchen Fuss man ergreift.

Beabsichtigt man, dass das heruntergeholte und ausgestreckte Bein unmittelbar nach vollzogener Wendung vorn, hinter der Symphyse liegen soll, so wird man in der Mehrzahl der Fälle dieses erreichen, wenn man bei dorso-anterioren Lagen das untere (vergleiche jedoch das Seite 5 Gesagte) bei dorso-posterioren das obere Bein (siehe das Seite 3 Gesagte) herunterholt.

Hat man indessen durch die Wendung eine unvollkommene Fusslage mit ausgestrecktem hinteren Bein hergestellt, so wird letzteres sich in der Weise nach vorne drehen, dass der Rücken des Kindes dem Promontorium vorbeigleitet.

Sieht man sich in Fällen der letztgenannten Art genöthigt, die Geburt des Kindes za beschleunigen, so muss man die Drehung des kindlichen Rumpfes um seine Längsachse, damit das ausgestreckte Bein nach vọrn kommt, in obigem Sinne begünstigen. 